\title{
A Spinal Muscular Atrophy Family with Intrafamilial Phenotype Differences Despite the Same Copy-Number Variation in SMN2
}

\author{
Jin-Mo Park ${ }^{a}$ \\ Hisahide Nishio ${ }^{b}$ \\ Jin-Hong Shin ${ }^{c}$ \\ Jin-Sung Park ${ }^{\mathrm{d}, \mathrm{e}}$ \\ aDepartment of Neurology, \\ Dongguk University College of Medicine, \\ Gyeongju, Korea \\ ${ }^{b}$ Department of Community Medicine and \\ Social Healthcare Science, \\ Kobe Univiersity Graduate \\ School of Medicine, Kobe, Japan \\ 'Department of Neurology, \\ Pusan National University \\ Yangsan Hospital, Yangsan, Korea \\ 'Department of Neurology, \\ School of Medicine, \\ Kyungpook National University, \\ Daegu, Korea \\ Department of Neurology, \\ Kyungpook National University \\ Chilgok Hospital, Daegu, Korea
}

Received November 20, 2018

Revised December 22, 2018

Accepted December 27, 2018

\section{Correspondence}

Jin-Sung Park, MD

Department of Neurology,

School of Medicine

Kyungpook National University,

680 Gukchaebosang-ro, Jung-gu,

Daegu 41944, Korea

Tel +82-53-200-2753

Fax +82-53-200-2029

E-mailnneurojspark@gmail.com
Dear Editor,

Spinal muscular atrophy (SMA) is caused by homozygous deletion of the survival motor neuron gene (SMN1), and copy-number variation (CNV) in the SMN2 gene is thought to influence the disease severity. ${ }^{1}$ We present two late-onset SMA siblings who manifested with marked differences in clinical severity and muscle imaging despite the same copy numbers of SMN2.

A 43-year-old female presented with proximal limb weakness since high school that had not deteriorated until she reached 40 years of age. The initial neurological examination of the proband demonstrated proximal limb weakness in the lower extremities, at MRC grade 4. However, she had no difficulty in performing the activities of daily living, but experienced difficulties during strenuous exercise such as hiking. The proband's elder brother was 45 years old, and he had found it difficult to run during high school, and had been incapable of independent walking since his 30s. The initial neurological examination revealed a proximal limb weakness with the lower extremity being affected more, and he was nonambulatory. Muscle MRI revealed that the proximal thigh muscles were markedly affected with relative sparing of the adductor and gracilis muscles (Fig. 1A). Her muscle biopsy showed fiber-type grouping, implicating neurogenic changes (Supplementary Fig. 1 in the online-only Data Supplement). The muscle MRI of the proband's elder brother illustrated a diffuse fatty change in proximal muscles including gluteus maximus, gluteus medius vastus, and hamstring muscles, but the gracilis and adductor longus muscles were mildly spared (Fig. 1B). A gene study of SMN1 revealed deletion of exons 7 and 8, confirming the diagnosis of SMA; the proband was classified as SMA4, while it was more appropriate to classify her brother as SMA3b. We measured the copy number of SMN2 of these two siblings using two different methods to ensure a precise diagnosis. We compared the SMN2, NAIP, and CFTR copy numbers as described previously, ${ }^{1}$ and we additionally used droplet digital PCR analysis (Bio-Rad Laboratories; ddPCR SMN2 copy number determination kit, Hercules, CA, USA). The obtained results were further confirmed by multicopy marker analysis as described by Melki et al. ${ }^{2}$ Both patients showed four copies of SMN2 and the same NAIP and CFTR copy numbers. Next-generation sequencing was also performed to screen the variants in $D Y N C 1 H 1$, BICD2, SMN1, PLS3, and NCALD, but this revealed no pathogenic mutation or known modifier known to affect the phenotype of our patients.

The CNVs of SMN2 are generally known to be correlated with the phenotype. ${ }^{1,3-5}$ However, there are reports of phenotype discrepancies in patients with same copy numbers of SMN2.,5 This means that variability in the phenotype cannot be reliably explained only by CNVs, and so we expect that there are other unknown genetic modifiers, sex differences, or epigenetic factors. It has reported that there are sex-related differences in severity or in intra-

@ This is an Open Access article distributed under the terms of the Creative Commons Attribution Non-Commercial License (https://creativecommons.org/licenses/by-nc/4.0) which permits unrestricted non-commercial use, distribution, and reproduction in any medium, provided the original work is properly cited. 

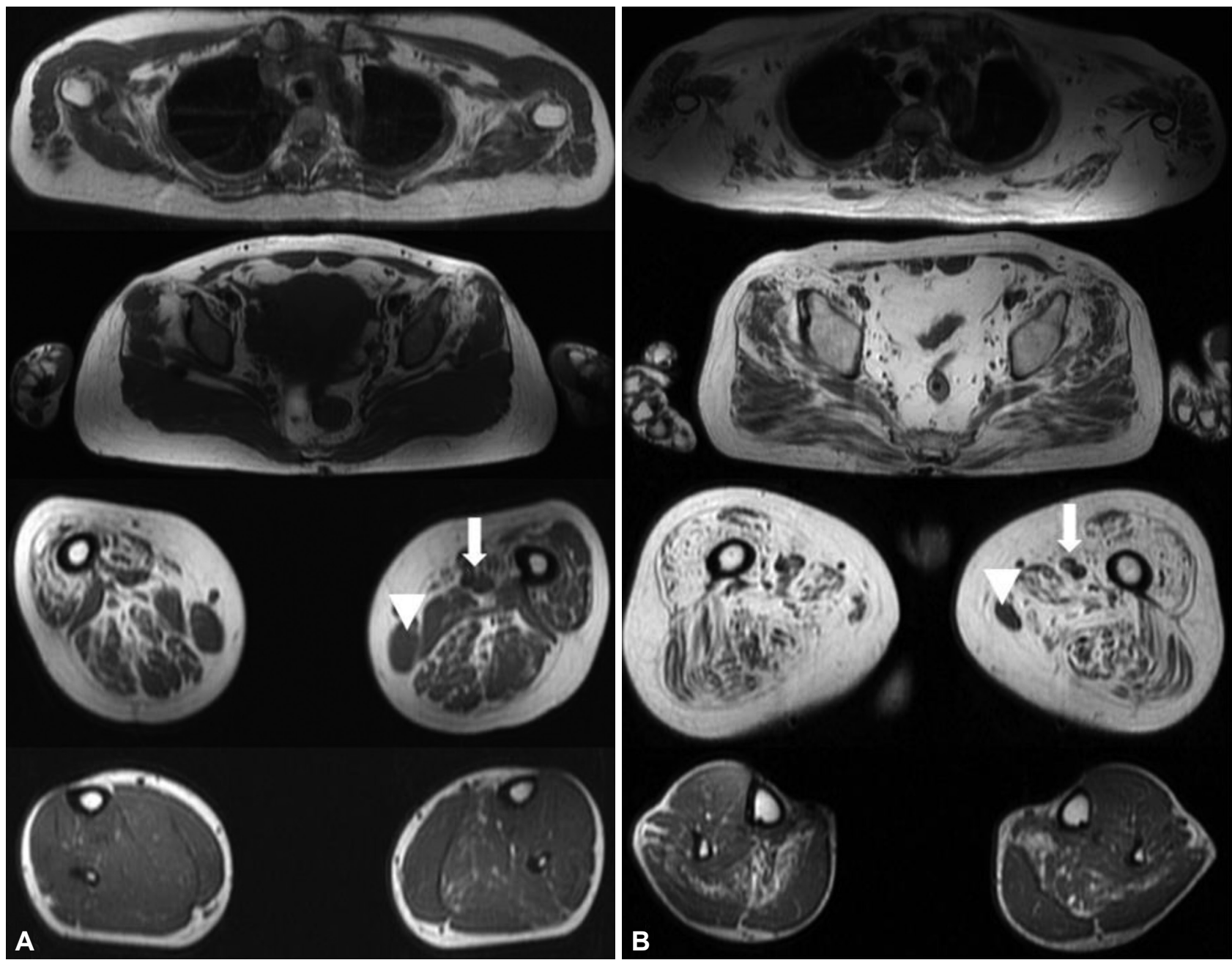

Fig. 1. Muscle MRI findings for the proband (A) and her elder brother (B). The images show selective involvement of muscles with relatively spared involvement in the adductor longus (arrows) and gracilis (arrowheads) muscles.

familial phenotype variability. ${ }^{4,6-8}$ A recent clinical study of myotonic dystrophy type 1 showed that sex differences might be another modifying factor influencing the clinical profile and severity of the disease. ${ }^{6}$ Consistent with these studies, we observed a significantly more severe phenotype in the male patient but a mild phenotype in the female patient of the same family. Therefore, even in SMA, sex difference is a possible modifying factor associated with phenotype variability, and further studies are warranted to clarify this.

\section{Supplementary Materials}

The online-only Data Supplement is available with this article at https://doi.org/10.3988/jen.2019.15.3.395.

\section{Conflicts of Interest}

The authors have no potential conflicts of interest to disclose.

\section{Acknowledgements}

This work was supported by the National Research Foundation of Korea
(NRF) grants funded by the Korea government (Ministry of Science and ICT) (Grant Nos. 2017R1C1B5076264 and NRF-2018R1C1B5045675).

\section{REFERENCES}

1. Tran VK, Sasongko TH, Hong DD, Hoan NT, Dung VC, Lee MJ, et al. SMN2 and NAIP gene dosages in Vietnamese patients with spinal muscular atrophy. Pediatr Int 2008;50:346-351.

2. Melki K, Lefebvre S, Burglen L, Burlet P, Clermont O, Millasseau P, et al. De novo and inherited deletions of the $5 \mathrm{q} 13$ region in spinal muscular atrophies. Science 1994;264:1474-1477.

3. Durmus H, Yilmaz R, Gulsen-Parman Y, Oflazer-Serdaroglu P, Cuttini $M$, Dursun $M$, et al. Muscle magnetic resonance imaging in spinal muscular atrophy type 3 : selective and progressive involvement. Muscle Nerve 2017;55:651-656.

4. Feldkötter M, Schwarzer V, Wirth R, Wienker TF, Wirth B. Quantitative analyses of SMN1 and SMN2 based on real-time LightCycler PCR: fast and highly reliable carrier testing and prediction of severity of spinal muscular atrophy. Am J Hum Genet 2002;70:358-368.

5. Wirth B, Herz M, Wetter A, Moskau S, Hahnen E, Rudnik-Schöneborn $S$, et al. Quantitative analysis of survival motor neuron copies: identification of subtle SMN1 mutations in patients with spinal muscular atrophy, genotype-phenotype correlation, and implications for genetic 
atrophy: the number of SMN2 gene copies, deletion in the NAIP gene and probably gender influence the course of the disease. Acta Biochim Pol 2009;56:103-108.

8. Cuscó I, Barceló MJ, Rojas-García R, Illa I, Gámez J, Cervera C, et al. SMN2 copy number predicts acute or chronic spinal muscular atrophy but does not account for intrafamilial variability in siblings. J Neurol 2006;253:21-25. 